\title{
Towards wafer-scale integration of high repetition rate passively mode-locked surface-emitting semiconductor lasers
}

\author{
Journal Article \\ Author(s): \\ Lorenser, D.; Unold, H.J.; Maas, Deran; Aschwanden, A.; Grange, R.; Paschotta, R.; Ebling, D.; Gini, E.; Keller, U. \\ Publication date: \\ 2004-12
}

Permanent link:

https://doi.org/10.3929/ethz-b-000038172

Rights / license:

In Copyright - Non-Commercial Use Permitted

Originally published in:

Applied Physics B 79(8), https://doi.org/10.1007/s00340-004-1675-3 


\author{
D. LORENSER ${ }^{1}$ \\ H.J. UNOLD ${ }^{1,-]}$ \\ D.J.H.C. MAAS ${ }^{1}$ \\ A. ASCHWANDEN ${ }^{1}$ \\ R. GRANGE ${ }^{1}$ \\ R. PASCHOTTA ${ }^{1}$ \\ D. EBLING $^{2}$ \\ E. GINI ${ }^{2}$ \\ U. KELLER ${ }^{1}$
}

\section{Towards wafer-scale integration of high repetition rate passively mode-locked surface-emitting semiconductor lasers}

\footnotetext{
${ }^{1}$ Institute of Quantum Electronics, Physics Department, Swiss Federal Institute of Technology (ETH), ETH Zürich Hönggerberg, Wolfgang-Pauli-Str. 16, 8093 Zürich, Switzerland

${ }^{2}$ FIRST Center for Micro- and Nanoscience, Swiss Federal Institute of Technology (ETH), ETH Zürich Hönggerberg, Wolfgang-Pauli-Str. 10, 8093 Zürich, Switzerland
}

Received: 28 September 2004

Published online: 4 November 2004 • C Springer-Verlag 2004

ABSTRACT One of the most application-relevant milestones that remain to be achieved in the field of passively mode-locked surface-emitting semiconductor lasers is the integration of the semiconductor absorber into the gain structure, enabling the realization of ultra-compact high-repetition-rate laser devices suitable for wafer-scale integration. We have recently succeeded in fabricating the key element in this concept, a quantumdot-based saturable absorber with a very low saturation fluence, which for the first time allows stable mode locking of surface-emitting semiconductor lasers with the same mode areas on gain and absorber. Experimental results at high repetition rates of up to $30 \mathrm{GHz}$ are shown.

PACS 42.55.Px; 42.60.Fc; 42.82.Gw

1

\section{Introduction}

The optically pumped vertical-external-cavity surface-emitting semiconductor laser (VECSEL) is a laser source that has sparked widespread interest in the past few years due to its capability of producing high average output powers in a diffraction-limited beam [1]. Furthermore, the semiconductor gain medium is well suited for being passively mode-locked with a semiconductor saturable-absorber mirror (SESAM, [2,3]), and its large gain cross-section allows stable mode locking at multi-GHz repetition rates without Q-switching instabilities [4]. The first passively mode-locked VECSEL was demonstrated in the year 2000 in collaboration with our group, with an average output power of $22 \mathrm{~mW}$ [5]. In 2002 it was shown that, due to their large gain bandwidth, VECSELs are capable of producing pulses shorter than $500 \mathrm{fs}$ [6]. The milestone of nearly $1 \mathrm{~W}$ average output power was achieved in
2002 at a repetition rate of $6 \mathrm{GHz}$ [7]. The pulses in the early experiments were often strongly chirped, but in 2002 a study of the mode-locking dynamics in VECSELs revealed that a solitonlike pulse shaping mechanism in the positive dispersion regime can help to generate short pulses with low chirp [8]. With the aid of intracavity dispersion control, it became possible to obtain nearly transform-limited pulses with record-high output powers of $2.1 \mathrm{~W}$ at $4 \mathrm{GHz}$ [9] and $1.4 \mathrm{~W}$ at $10 \mathrm{GHz}$ [10].

Recently, a number of applications like optical clocking and telecommunications have created a demand for very-high-repetition-rate sources (tens of gigahertz) with high average powers (up to the watt range). Passively mode-locked VECSELs have the potential for meeting such specifications, and we believe that their further development should lead to commercially very attractive devices that can be produced with cost-effective wafer-scale technologies.
In the first section of this article, we discuss some general issues that need to be dealt with when pushing VECSELs to higher repetition rates in the range between 10 and $100 \mathrm{GHz}$. The obvious approach is to simply scale down the dimensions of the currently employed cavity concepts, and the limitations of this scheme will be analyzed. Then, in order to reach the highest possible repetition rates and to obtain less complicated and more stable cavities, we will discuss the possibility of merging the semiconductor gain and absorber into one monolithic structure that can be grown in a single epitaxy process. This would result in highly compact and simple devices suitable for low-cost wafer-scale mass production. It will be shown that the key element for both the conventional scaling-down approach as well as the integrated-absorber VECSEL is an absorber with a lower saturation fluence than that of the current state-of-the-art quantum well SESAMs (QW SESAM).

In Sect. 2 we present such an absorber, a SESAM incorporating quantum dots, and we discuss its characteristics and fabrication. We show experimental results at record-high repetition rates of $21 \mathrm{GHz}$ and $30 \mathrm{GHz}$ which demonstrate that this SESAM for the first time allows the mode locking of VECSELs with the same mode areas on gain and absorber, which is an important test for the feasibility of the integratedabsorber concept.

Finally, we draw conclusions from the obtained results and give an outlook on the further developments that can be anticipated after the positive outcome of these experiments. 


Towards higher repetition
rates and integrated-
absorber VECSELs

2.1

\section{Scaling down conventional external-absorber cavities}

The typical cavity for mode locking a VECSEL with a QW SESAM is shown in Fig. 1a. It has a folded V-shaped geometry and consists only of the SESAM as the end mirror, the gain structure as the folding mirror, and an output coupler with radius of curvature $R$. Other types of cavities are conceivable, for example linear geometries using an output-coupler SESAM [11] and an intracavity lens [12], but the issues discussed in the following will generally apply to them in a similar way. The cavity length $l_{\text {cav }}$ is adjusted close to the stability limit $\left(l_{\mathrm{cav}} \approx R\right.$ in our example) in order to obtain a strongly divergent mode which is focused tightly on the SESAM and which is larger on the gain. This is necessary because the pulse formation mechanism in VECSELs $[8,13,14]$ dictates that the absorber must saturate more strongly
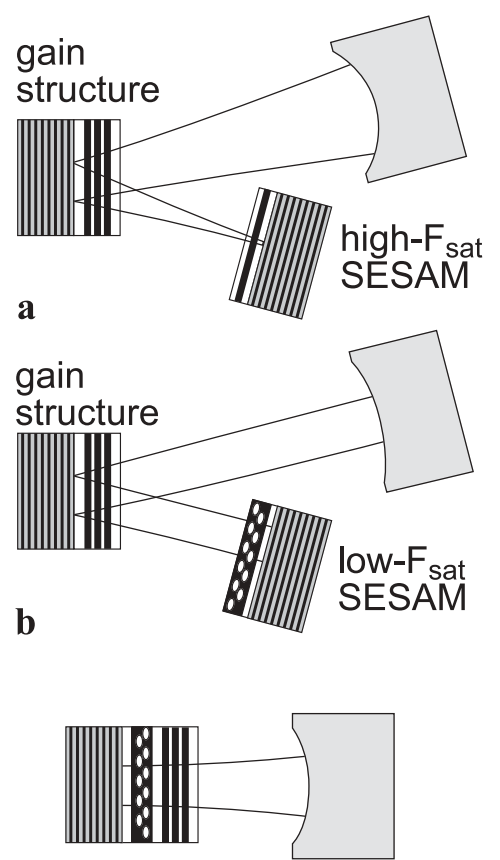

integrated-absorber VECSEL

c

FIGURE 1 Schematic illustrationn of the progression towards the integrated-absorber VECSEL. (a) Folded Cavity with high- $F_{\text {sat }}$ SESAM requiring a tight focus on the absorber. (b) Folded Cavity with low- $F_{\text {sat }}$ SESAM where mode sizes on gain and absorber can be equal. (c) Simple linear cavity with an integrated-absorber VECSEL than the gain. The saturation energies $E_{\mathrm{sat}, g}$ and $E_{\mathrm{sat}, a}$ of gain and absorber must therefore fulfill the following inequality:

$\frac{E_{\text {sat,a }}}{E_{\text {sat }, \mathrm{g}}}=\frac{F_{\text {sat }, \mathrm{a}} A_{\mathrm{a}}}{F_{\text {sat }, \mathrm{g}} A_{\mathrm{g}}} \ll 1$

where $F_{\text {sat, }}, F_{\text {sat,a }}$ and $A_{\mathrm{g}}, A_{\mathrm{a}}$ are the saturation fluences and mode areas of gain and absorber. Due to the fact that $F_{\text {sat,g }}$ and $F_{\text {sat,a }}$ are of the same order of magnitude, since both gain and absorber are based on quantum wells of usually the same material system, the mode area ratio $A_{\mathrm{g}} / A_{\mathrm{a}}$ typically needs to be adjusted to values between 10 and 30 for stable mode locking.

When going to high repetition rates, these cavities suffer from two problems. The first problem is that for a cavity of very short total length (e.g., $3 \mathrm{~mm}$ at $50 \mathrm{GHz}$ ) which is critically adjusted close to the stability limit, even small length changes due to mechanical vibrations or thermal drifts result in large changes of the mode areas on gain and absorber. Therefore these lasers become very sensitive to all kinds of perturbations and will not exhibit stable modelocking unless great care is exercised in the mechanical engineering of the resonator.

The second problem, which concerns the heating of the SESAM, is not such an obvious but nonetheless critical issue that becomes increasingly serious at higher repetition rates. The reason is that, for a given average power level, the energy per pulse decreases for increasing repetition rates, making it necessary to focus the cavity mode more and more tightly on the SESAM in order to maintain a constant level of saturation which is required for sustaining the mode locking. Therefore the same average absorbed power causes a higher temperature rise at higher repetition rates because it is deposited on smaller spots.

In the following we give some simple quantitative expressions which will serve to identify the critical quantities and to point out approaches for improvement and optimization. We shall consider a SESAM with saturation fluence $F_{\text {sat,a, }}$, modulation depth $\Delta R$ and nonsaturable losses $\Delta R_{\mathrm{ns}}$. It is mode locking a laser with a given average intracavity power $P_{\text {int }}$ at a repetition rate $f_{\text {rep }}$, which means that the energy per pulse is $E_{\mathrm{p}}=P_{\text {int }} / f_{\text {rep }}$. For stable mode locking we must also ensure that the SESAM is operated at a given saturation parameter $S>1$ which is defined as

$$
\begin{aligned}
S & =\frac{E_{\mathrm{p}}}{E_{\text {sat }, \mathrm{a}}}=\frac{P_{\text {int }}}{F_{\text {sat, } \mathrm{a}} A_{\mathrm{a}} f_{\text {rep }}} \\
& =\frac{P_{\text {int }}}{F_{\text {sat }, \mathrm{a}} \pi w_{\mathrm{a}}^{2} f_{\text {rep }}}
\end{aligned}
$$

where $w_{\mathrm{a}}$ is the $1 / \mathrm{e}^{2}$ radius of the Gaussian intensity distribution of the laser mode on the SESAM. We assume that a heating power $P_{\text {heat }}$ with an associated transverse intensity distribution identical to that of the intensity of the laser mode is absorbed in a thin region near the surface of the SESAM (in the absorber and possibly also in the adjacent spacers and the mirror due to nonsaturable losses) and that it is then dissipated into an infinite half-space of substrate material with a thermal conductivity $\kappa$. This is a valid approximation for substrates thicker than $3 w_{\mathrm{a}}$ [7], and in this context we are dealing with laser mode radii $w_{\mathrm{a}}$ of $30 \mu \mathrm{m}$ or less and substrate thicknesses of $300 \mu \mathrm{m}$ or more. Under these conditions, the temperature rise $\Delta T$ in the center of the spot with reference to the ambient or heat-sink temperature is [15]:

$$
\Delta T=\frac{P_{\text {heat }}}{\sqrt{2 \pi} w_{\mathrm{a}} \kappa} .
$$

For the approximation of weak absorption (a few percent, as is typically the case in the absorbers discussed here), it can be shown that the absorbed average power in the SESAM is [15]:

$$
P_{\text {heat }}=P_{\text {int }}\left(\Delta R_{\mathrm{ns}}+\frac{\Delta R}{S}\left[1-\mathrm{e}^{-S}\right]\right) .
$$

The first term in the brackets on the right side simply accounts for the power dissipated in the nonsaturable losses, while the second term results from the absorbed pulse energy per round-trip which is necessary for bleaching the absorber. Solving (2) for $w_{\mathrm{a}}$ and inserting this into (3) together with (4) yields

$$
\begin{aligned}
\Delta T= & \frac{1}{\sqrt{2} \kappa} \sqrt{P_{\text {int }} f_{\text {rep }} F_{\text {sat }, \mathrm{a}} S} \\
& \times\left(\Delta R_{\mathrm{ns}}+\frac{\Delta R}{S}\left(1-\mathrm{e}^{-S}\right)\right) .
\end{aligned}
$$


This is an approximation of the temperature rise in the SESAM which in reality will show small heating transients occurring at the pulse repetition rate. Simplified analytical solutions of the heat equation show that these transients superimposed on the time-average given by (5) are on the order of only a few Kelvins at the operating conditions relevant to this context, so the approximation is valid. The essential implication of (5) is that, for a given power level and given SESAM parameters, the temperature rise increases with the square root of the repetition rate.

In high-power mode-locking experiments at repetition rates between 1 and $10 \mathrm{GHz}$ we have observed that the maximum output power was often limited by SESAM damage. When calculating the SESAM temperature rise in these cases with (5) we obtain values of around $80 \mathrm{~K}$. This is not a temperature rise at which semiconductors typically exhibit damage, and the reasons for this relatively low failure-temperature are not yet fully understood. One possible explanation is that this value represents a kind of threshold temperature rise at which SESAM properties degrade to a point where a thermal runaway situation could begin to set in. This is supported by the fact that three important quantities in (5) change in a detrimental way for increasing temperatures: the thermal conductivity $\kappa$ of the $\mathrm{Al}_{x} \mathrm{Ga}_{1-x} \mathrm{As}$ material system decreases and also the bandgap energy of the absorber decreases, increasing $\Delta R$ and $F_{\text {sat,a }}$ (because the carriers are excited higher into the band). These effects can create a positive-feedback mechanism for the heating, and the temperature at which the SESAM is actually damaged could then be much higher.

From (5) we can now also roughly estimate the maximum power that we can obtain at higher repetition rates from VECSELs with QW SESAMs in cavities operated at the stability limit. With our current VECSEL devices and SESAMs, the maximum power that could be extracted at $4 \mathrm{GHz}$ was $2.1 \mathrm{~W}$ [9]. Keeping the product $P_{\text {int }} f_{\text {rep }}$ constant and assuming that we use the same output coupling, the maximum power that we can obtain with this SESAM at $40 \mathrm{GHz}$ would therefore be around $200 \mathrm{~mW}$.
Even for high-quality SESAMs with minimized nonsaturable losses, (5) shows that the possibilities of further reducing the heating are limited. Reducing $\Delta R$ and $S$ to the lowest values that still ensure stable mode locking or reducing the intracavity power $P_{\text {int }}$ by using more output coupling only offers small margins for improvement. A more significant performance gain can be expected from reducing the thermal impedance of the SESAM by substrate removal and soldering to a heat spreader material with higher thermal conductivity, thereby increasing the effective value of $\kappa$.

The real high-leverage quantity in (5), however, is the saturation fluence $F_{\text {sat,a }}$. Employing absorber materials with substantially lower $F_{\text {sat }}$ will effectively counteract both problems that have been outlined above. Not only does the lower $F_{\text {sat }}$ directly reduce the temperature rise and therefore permit an increase of the power level by the same factor, it also allows much larger mode radii $w_{\mathrm{a}}$ for the same pulse energy while maintaining the desired saturation parameter $S$ of the SESAM, as can be seen from (2). This eliminates the problem of having to operate the cavity close to its stability limit and therefore makes it possible to design stable and rugged resonators suitable for real-world applications. By making use of low- $F_{\text {sat }}$ SESAMs, the conventional externalabsorber cavities can therefore be expected to be down-scalable all the way to the practical limits of mechanical miniaturization while maintaining reasonably high average output powers.

\subsection{The final step: integration of the absorber}

Scaling down VECSELs to the highest possible repetition rates naturally leads one to contemplate some form of monolithic structure in which gain and absorber are integrated into one single semiconductor layer sequence. Such a monolithic device would allow one to reduce the cavity to a simple linear geometry requiring only an external output coupler at a distance from the chip which determines the desired repetition rate. The basic obstacle which has prevented the realization of such a device so far has been the lack of a suitable absorber material which has a sufficiently low saturation fluence in order to fulfill (1) with equal mode sizes on gain and absorber. The recent development of low- $F_{\text {sat }}$ SESAMs based on a quantum-dot absorber material, which will be discussed in the next section, has made it realistic to move towards this goal along the scheme shown in Fig. 1. As we have explained above, reducing $F_{\text {sat }}$ will allow one to gradually move away from cavities that focus the mode very tightly on the absorber as in Fig. 1a. If $F_{\text {sat }}$ can be reduced to the point where mode locking can be achieved in cavities like the one depicted in Fig. 1b, which have the same mode sizes on absorber and gain, one can then take the final step and integrate the absorber into the gain structure in order to arrive at our desired configuration shown in Fig. 1c. By systematic optimization of low- $F_{\text {sat }}$ quantum-dot SESAMs, we have recently succeeded in arriving at the stage of development represented by Fig. 1b, which is the crucial go/nogo experiment that must work before an integration of the absorber can be attempted.

When designing such an integrated device, attention has to be paid to some consequences of having gain and absorber in the same semiconductor structure: firstly, both components act as heat sources, which makes good thermal management even more important. Also, the absorber will most likely need to be protected from the pump light incident on the semiconductor chip. Therefore, the absorber should be located beneath the gain structure and separated by a pump-reflecting mirror. This intermediate mirror can simultaneously serve as a means to adjust the amount of optical resonance at the laser wavelength in the gain and absorber sections necessary for stable mode locking. Finally, for the ultimate state of integration, one could envisage a monolithic structure needing no external inputs except an electric power source. Such a device would involve integrating the output coupler onto the semiconductor chip [16] and pumping the gain electrically [17].

3 Successful pre-integration experiments at 21 and $30 \mathrm{GHz}$

3.1 The low-F sat $_{\text {SESAM }}$

The SESAM used in the experiments described in the following is 
realized as a resonant design at $955 \mathrm{~nm}$. It contains a 30-pair AlAs/GaAs DBR and a single absorber layer embedded in GaAs and placed in an antinode of the standing wave pattern. In order to obtain a low $F_{\text {sat }}$, self-assembled InAs quantum dots (QDs) are used in the absorber layer. Due to their three-dimensional carrier confinement, the density of states tion, which in turn results in a strongly reduced saturation fluence compared to quantum wells [18]. The structure is grown by solid-source molecular beam epitaxy (MBE) at $580{ }^{\circ} \mathrm{C}$ with the exception of the QDs, which are grown at a temperature of $380^{\circ} \mathrm{C}$, which introduces crystal defects acting as recombination centers and thereby enabling fast absorber recovery times. The QDs are formed by delivering 1.8 monolayers of InAs to the sample, yielding a photoluminescence (PL) peak wavelength and FWHM of $958 \mathrm{~nm}$ and $36 \mathrm{~nm}$, respectively. Figure 2 displays a saturation fluence measurement of the QD SESAM [19] of a wavelength of $960 \mathrm{~nm}$. The measurement was done with 290-fs pulses; therefore, the curve shows a rollover at high fluences due to two-photon absorption (TPA). The extrapolated fit curve without TPA is also plotted because it gives a better impression of the absorber behavior in the picosecondpulsewidth domain in which it is later operated in the laser [20]. The fit yields $F_{\text {sat }}=1.7 \mu \mathrm{J} / \mathrm{cm}^{2}$, which is an order of magnitude lower than the typical values of around $20 \mu \mathrm{J} / \mathrm{cm}^{2}$ that can be is ideally compressed into a delta-func-

achieved with optimized QW SESAMs at this wavelength. The modulation depth $\Delta R$ is $3.1 \%$ and the nonsaturable losses $\Delta R_{\mathrm{ns}}$ are $0.3 \%$.

\subsection{Experimental results: VECSELS with 21 and $30 \mathrm{GHz}$}

The QD SESAM was installed in the cavity setup shown in Fig. 3. The total cavity length was $7.1 \mathrm{~mm}$ at $21 \mathrm{GHz}$ and was later reduced to $5 \mathrm{~mm}$ for a repetition rate of $30 \mathrm{GHz}$. An output coupler with a large radius of curvature of $200 \mathrm{~mm}$ was used, resulting in a very weakly focused mode that had practically identical mode radii of about $90 \mu \mathrm{m}$ on the gain and the absorber. An output coupler transmission of only $0.35 \%$ had to be used (compared to $2.5 \%$ as a typical value for our mode-locked VECSELs) because the SESAM had high small-signal losses due to the large modulation depth which was higher than desired. The output powers that could be extracted were therefore quite low and are not representative of the high-power capabilities of VECSELs. The pump source was a commercial 808-nm diode module capable of delivering up to $4.2 \mathrm{~W}$ in a homogenized beam with the same $M^{2}$ of about 13 in both axes, focused down to a nearly circular pump spot with radii of $92 \mu \mathrm{m}$ and $85 \mu \mathrm{m}$. A $20-\mu \mathrm{m}$ uncoated fused-silica etalon was used for tuning the laser wavelength into regions where the total intracavity group delay dispersion (GDD) is expected to be positive,

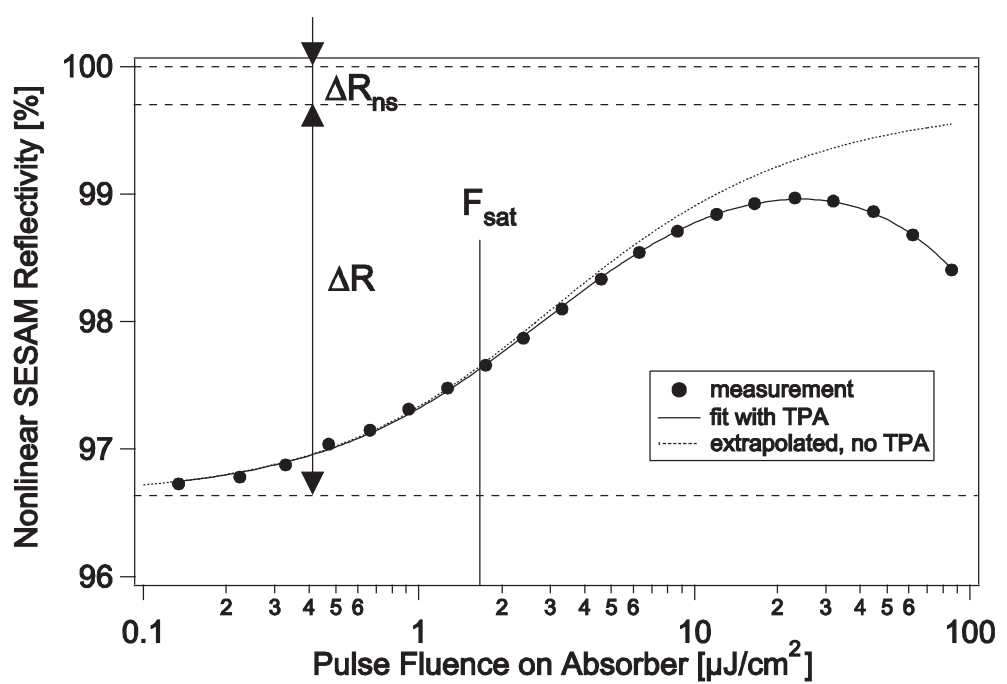

FIGURE 2 Saturation fluence measurement and fitted curves for the quantum-dot SESAM used in the mode locking experiments which has been shown to be favorable for obtaining stable mode locking and short pulses $[8,9]$.

The two VECSEL gain structures used in these experiments are of similar design and consist of three parts: a highly reflecting bottom mirror, the active region, and an anti-reflective (AR) section. The AlAs $/ \mathrm{Al}_{0.2} \mathrm{Ga}_{0.8}$ As bottom mirror is optimized for high reflectivity at the pump $(808 \mathrm{~nm})$ and laser wavelength $(950$ to $980 \mathrm{~nm})$ for an incident angle of $45^{\circ}$ and normal incidence, respectively. Most of the pump light is absorbed in the active region, which consists of seven QWs separated by spacer sections made up of pump-absorbing layers and strain-compensating layers. The $\operatorname{In}_{0.13} \mathrm{Ga}_{0.87}$ As quantum wells are placed in the antinodes of the standingwave pattern. The pump-absorbing layers consist of $\mathrm{Al}_{0.06} \mathrm{Ga}_{0.94} \mathrm{As}$ in the first structure, and GaAs in the second structure. The strain-compensating layers consist of $\mathrm{GaAs}_{0.94} \mathrm{P}_{0.06}$, which also absorbs at the pump wavelength of $808 \mathrm{~nm}$. They are positioned directly adjacent to the QWs on both sides in the first structure, whereas they are placed in the center of the spacer sections in the second structure. The semiconductor layer sequence is grown in reverse order with metal-organic vapor phase epitaxy (MOVPE) and soldered to a copper heatsink. The substrate is then removed, resulting in a gain structure with minimized thermal impedance. Details on the growth and processing can be found in [7].

The first gain structure, which lases at long wavelengths around $980 \mathrm{~nm}$, was chosen to avoid the high losses in the vicinity of the SESAM resonance at $955 \mathrm{~nm}$. From the post-growth characterization of this gain structure, the

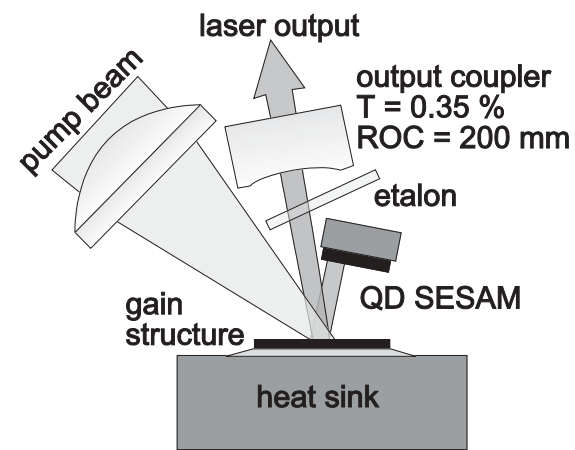

FIGURE 3 Cavity setup used for the modelocking experiments at 21 and $30 \mathrm{GHz}$ 
GDD was calculated to be positive in the wavelength interval between 958 and $977 \mathrm{~nm}$ (We estimate that these values can have errors in the order of $2 \mathrm{~nm}$ ). With the etalon we were able to tune the wavelength from 975 to $981 \mathrm{~nm}$ and mode locking was possible over this entire range, with only a weak trend towards shorter pulses at shorter wavelengths, however, with lower output powers because of the proximity to the SESAM resonance. Figure 4 shows the pulse characterization of our best result. We were able to obtain $55 \mathrm{~mW}$ of average output power at a repetition rate of $21 \mathrm{GHz}$. The incident pump power was $2.5 \mathrm{~W}$ and the heatsink of the gain structure was held at $30^{\circ} \mathrm{C}$. The autocorrelation can be fitted well with an ideal $\mathrm{sech}^{2}$ pulse of $9.7 \mathrm{ps}$ duration. The optical spectrum is centered at $980.3 \mathrm{~nm}$ with a FWHM of $0.33 \mathrm{~nm}$. At the time of the experiment only a photodiode with an approximate 3 -dB bandwidth of $17 \mathrm{GHz}$ was available, so the RF spectrum does not have a very good signal to noise ratio. However, the wide-scan autocorrelation shows a stable $21-\mathrm{GHz}$ pulse train with clearly separated pulses. The time-bandwidth product of 1.0 is 3.2 times above the transform limit, indicating that the pulses are moderately chirped.

The second gain structure which lases at shorter wavelengths around $955 \mathrm{~nm}$ was then installed in the cavity. Even though this is right at the SESAM resonance, it was possible to get the VECSEL to lase and to tune it over the range of 947 to $960 \mathrm{~nm}$. The GDD of this structure was calculated to be positive in the range from 940 to $957 \mathrm{~nm}$ (again with an uncertainty of about $2 \mathrm{~nm}$ ). Mode locking was only observed in the wavelength range 958 to $960 \mathrm{~nm}$. By optimizing the cavity length we were able to increase the repetition rate to $30 \mathrm{GHz}$. Our best result with an average output power of $25 \mathrm{~mW}$ is shown in Fig. 5. The incident pump power was $2.9 \mathrm{~W}$ and the heatsink of the gain structure was held at $16^{\circ} \mathrm{C}$. The autocorrelation can be fitted well with an ideal $\operatorname{sech}^{2}$ pulse of $4.7 \mathrm{ps}$ duration. The optical spectrum is centered at $959.6 \mathrm{~nm}$ with a FWHM of $0.31 \mathrm{~nm}$. The insufficient bandwidth of the photodiode again limits the signal to noise ratio of the RF spectrum, but the wide-scan autocorrelation shows a clean $30-\mathrm{GHz}$ pulse train with clearly sepa-
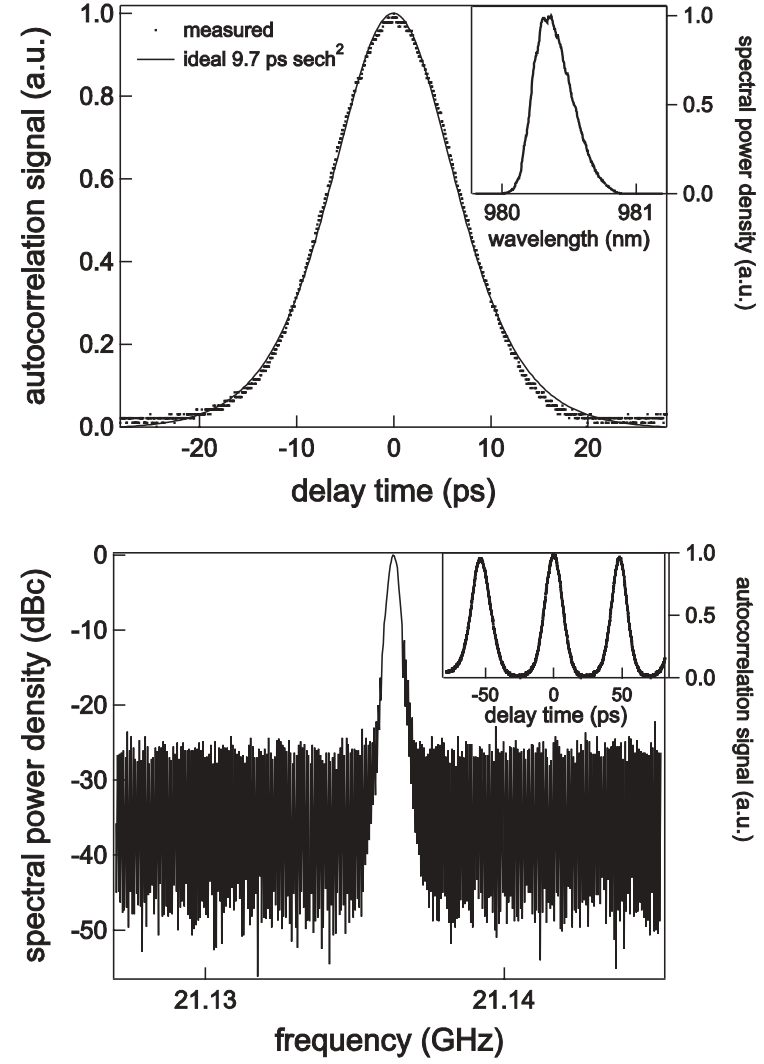

FIGURE 4 Measurement data of the 21-GHz result with $55 \mathrm{~mW}$ average output power. Top: Autocorrelation of the pulses. The inset shows the optical spectrum. Bottom: RF spectrum on a $20 \mathrm{MHz}$ span and with $300 \mathrm{kHz}$ resolution bandwidth. The inset shows a wide-scan autocorrelation of the pulse train
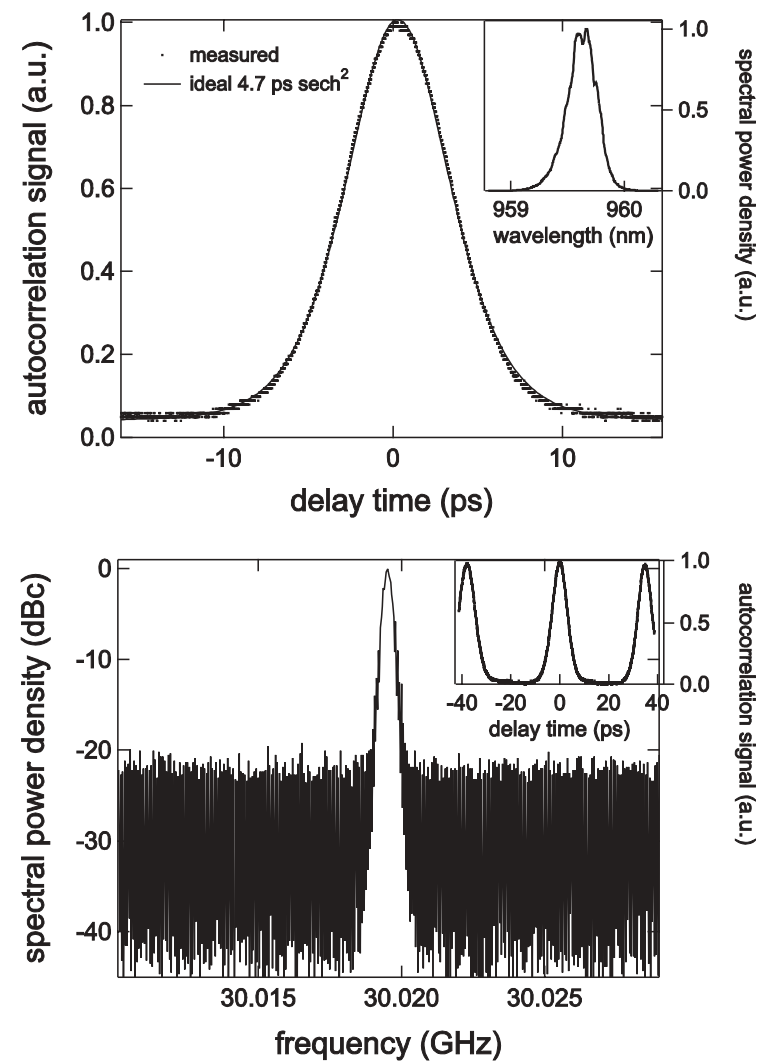

FIGURE 5 Measurement data of the $30-\mathrm{GHz}$ result with $25 \mathrm{~mW}$ average output power. Top: Autocorrelation of the pulses. The inset shows the optical spectrum. Bottom: RF spectrum on a $20 \mathrm{MHz} \mathrm{span}$ and with $300 \mathrm{kHz}$ resolution bandwidth. The inset shows a wide-scan autocorrelation of the pulse train 
rated pulses. The pulse quality is good with a time-bandwidth product of 0.5 , which is only 1.6 times above the transform limit.

\section{Conclusions and outlook}

We have given an overview of the challenges involved in progressing towards higher repetition rates with passively mode-locked VECSELs. We have shown that low- $F_{\text {sat }}$ SESAMs are the key for maintaining high average powers at high repetition rates with VECSELs due to limitations imposed by thermal issues and cavity-stability problems when employing conventional QW SESAMs. We would like to point out that these issues are not specific to VECSELs, and therefore other kinds of mode-locked lasers can also be expected to experience a noticeable performance boost with the advent of low$F_{\text {sat }}$ SESAMs.

Furthermore, we have discussed the possibility of integrating these low- $F_{\text {sat }}$ absorbers into the gain structure. A low$F_{\text {sat }}$ SESAM based on quantum-dot absorber material has been presented which enabled us to demonstrate for the first time the mode locking of VECSELs in a cavity configuration with the same mode areas on gain and absorber, which proves the feasibility of the integrated-absorber VECSEL concept. The fast recovery time of this SESAM allowed us to mode-lock at 21 and $30 \mathrm{GHz}$, showing that this absorber material is suitable for very high repetition rates. In fact, the repetition rate was not limited by the absorber characteristics but rather by the mechanical boundary conditions imposed by the dimensions of the cavity components and the optical pumping. The result at
$30 \mathrm{GHz}$ is to our knowledge the highest repetition rate obtained to date from this kind of laser in clean cw-mode-locked operation. The low- $F_{\text {sat }}$ QD SESAM still exhibits a rather large modulation depth as well as fairly high nonsaturable losses and will need further optimization in order to exploit the full potential of VECSELs in terms of output power.

If the remaining challenges posed by the design and the epitaxial growth of integrated-absorber VECSEL structures can be mastered, this concept will finally make wafer-scale mass production technology accessible to the field of passively mode-locked surface-emitting lasers. In particular, if electrical pumping of such devices can be realized, this will result in a new class of lowcost ultra-compact high-repetition-rate lasers with potentially high average output powers of more than $100 \mathrm{~mW}$ which will open the door to a range of applications which are currently not accessible with mode-locked edge-emitting laser diodes due to their very low output powers in the milliwatt range.

ACKNOWLEDGEMENTS We would like to acknowledge the kind assistance of Dr. M. Haiml who contributed to our understanding of SESAM heating issues in many helpful discussions. The authors are grateful for financial support from ETH Zurich and from an Intelsponsored research agreement.

\section{REFERENCES}

1 F. Kuznetsov, F. Hakimi, R. Sprague, A. Mooradian: IEEE Photon. Technol. Lett. 9, 1063 (1997)

2 U. Keller, D.A.B. Miller, G.D. Boyd, T.H. Chiu, J.F. Ferguson, M.T. Asom: Opt. Lett. 17, 505 (1992)

3 U. Keller, K.J. Weingarten, F.X. Kärtner, D. Kopf, B. Braun, I.D. Jung, R. Fluck, C. Hönninger, N. Matuschek, J. Aus der Au: IEEE J. Sel. Top. Quantum Electron. 2, 435 (1996)
4 C. Hönninger, R. Paschotta, F. MorierGenoud, M. Moser, U. Keller: J. Opt. Soc. Am. B 16, 46 (1999)

5 S. Hoogland, S. Dhanjal, A.C. Tropper, S.J. Roberts, R. Häring, R. Paschotta, U. Keller: IEEE Photon. Technol. Lett. 12, 1135 (2000)

6 A. Garnache, S. Hoogland, A.C. Tropper, I. Sagnes, G. Saint-Girons, J.S. Roberts: Appl. Phys. Lett. 80, 3892 (2002)

7 R. Häring, R. Paschotta, A. Aschwanden, E. Gini, F. Morier-Genoud, U. Keller: IEEE J. Quantum Electron. QE-38, 1268 (2002)

8 R. Paschotta, R. Häring, U. Keller, A. Garnache, S. Hoogland, A.C. Tropper: Appl. Phys. B 75, 445 (2002)

9 A. Aschwanden, D. Lorenser, H.J. Unold, R. Paschotta, E. Gini, U. Keller: submitted to Opt. Lett. (2004)

10 A. Aschwanden, D. Lorenser, H.J. Unold, R. Paschotta, E. Gini, U. Keller: Conference on Lasers and Electro-Optics (CLEO), San Francisco (USA), 2004, postdeadline paper CPDB8

11 G.J. Spühler, S. Reffert, M. Haiml, M. Moser, U. Keller: Appl. Phys. Lett. 78, 2733 (2001)

12 K. Jasim, Q. Zhang, A.V. Nurmikko, E. Ippen, A. Mooradian, G. Carey, W. Ha: Electron. Lett. 40, 34 (2004)

13 G.H.C. New: Opt. Commun. 6, 188 (1972)

14 H.A. Haus: IEEE J. Quantum Electron. QE-11, 736 (1975)

15 R. Paschotta, J. Aus der Au, G.J. Spühler, F. Morier-Genoud, R. Hövel, M. Moser, S. Erhard, M. Karszewski, A. Giesen, U. Keller: Appl. Phys. B 70, S25 (2000)

16 J.E. Hastie, J.-M. Hopkins, C.W. Jeon, S. Calvez, D. Burns, M.D. Dawson, R. Abram, E. Riis, A.I. Ferguson, W.J. Alford, T.D. Raymond, A.A. Allerman: Electron. Lett. 39, 1324 (2003)

17 J.G. McInerney, A. Mooradian, A. Lewis, A.V. Shchegrov, E.M. Strzelecka, D. Lee, J.P. Watson, M. Liebman, G.P. Carey, B.D. Cantos, W.R. Hitchens, D. Heald: Electron. Lett. 39, 523 (2003)

18 H. Shoji: "Self-Assembled Quantum Dot Lasers", In Self-Assembled InGaAs/GaAs Quantum Dots (Academic Press, San Diego 1999)

19 M. Haiml, R. Grange, U. Keller: Appl. Phys. B 79, 331 (2004)

20 R. Grange, M. Haiml, R. Paschotta, G.J. Spühler, L. Krainer, M. Golling, O. Ostinelli, U. Keller: Appl. Phys. B published online (2004) 